\title{
ROJ
}

\section{Extracranial systemic antitumor response through the abscopal effect induced by brain radiation in a patient with metastatic melanoma}

\author{
Mark A. D'Andrea, G. K. Reddy \\ University Cancer and Diagnostic Centers, Houston, TX, USA
}

The abscopal effect is a term that has been used to describe the phenomenon in which localized radiation therapy treatment of a tumor lesion triggers a spontaneous regression of metastatic lesion(s) at a non-irradiated distant site(s). Radiation therapy induced abscopal effects are believed to be mediated by activation and stimulation of the immune system. However, due to the brain's distinctive immune microenvironment, extracranial abscopal responses following cranial radiation therapy have rarely been reported. In this report, we describe the case of 42-year-old female patient with metastatic melanoma who experienced an abscopal response following her cranial radiation therapy for her brain metastasis. The patient initially presented with a stage III melanoma of the right upper skin of her back. Approximately 5 years after her diagnosis, the patient developed a large metastatic lesion in her upper right pectoral region of her chest wall and axilla. Since the patient's tumor was positive for BRAF and MEK, targeted therapy with dabrafenib and trametinib was initiated. However, the patient experienced central nervous system (CNS) symptoms of headache and disequilibrium and developed brain metastases prior to the start of targeted therapy. The patient received radiation therapy to a dose of $30 \mathrm{~Gy}$ delivered in 15 fractions to her brain lesions while the patient was on dabrafenib and trametinib therapy. The patient's CNS metastases improved significantly within weeks of her therapy. The patient's non-irradiated large extracranial chest mass and axilla mass also shrank substantially demonstrating the abscopal effect during her CNS radiation therapy. Following radiation therapy of her residual chest lesions, the patient was disease free clinically and her CNS lesions had regressed. However, when the radiation therapy ended and the patient continued her targeted therapy alone, recurrence outside of her previously treated fields was noted. The disease recurrence could be due to the possibility of developing BRAF resistance clones to the BRAF targeted therapy. The patient died eventually due to wide spread systemic disease recurrence despite targeted therapy.

Keywords: Abscopal effect, Brain metastasis, Central nervous system lesions, Immunostimulation, lonizing radiation, Molecular targeted therapy, Skin cancer

\section{Introduction}

Malignant melanoma represents the deadliest form of skin cancer. It has a high tendency to metastasize during the early course of the disease leading to death $[1,2]$. In fact, until relatively recently, treatment approaches for malignant melanoma had been extremely limited. However, advances in our understanding of its tumor biology have led to the use of

Received 26 July 2019, Revised 26 September 2019, Accepted 02 October 2019.

Correspondence: G. K. Reddy, University Cancer and Diagnostic Centers, 12811 Beamer Road. Houston, TX 77089, USA. Tel: +1-713-474-1414, Fax: +1-713-474-8477, E-mail: kreddy_usa@yahoo.com (https://orcid.org/0000-0002-1936-6972)

(c) This is an Open Access article distributed under the terms of the Creative Commons Attribution Non-Commercial License (http://creativecommons.org/ licenses/by-nc/4.0/) which permits unrestricted non-commercial use, distribution, and reproduction in any medium, provided the original work is properly cited.

www.e-roj.org 
novel treatment strategies aimed at eradicating the systemic disease. In recent years, several antitumor agents have been developed as an effective treatment to improve the overall survival of patients with metastatic melanoma [3-6].

Despite significant advances in the development of novel antitumor agents that improve overall survival, there is still a need for additional strategies to improve the treatment benefits in patients with metastatic malignant melanoma. Radiation therapy has long played a key role in the management of both local and metastatic melanoma. It exerts its antitumor effects by damaging the DNA that ultimately leads to tumor cell death. However, emerging evidence indicates that radiation therapy can also produce a systemic effect by stimulating the hosts' immune system $[7,8]$ and induce a phenomenon called 'the abscopal effect'. The term 'abscopal' was originally invented by Mole [9] in 1953 to describe a phenomenon whereby local irradiation of a tumor lesion triggers spontaneous regression of metastatic disease at a non-irradiated distant site. Localized radiation therapy has been shown to induce abscopal effects in several tumor types [10-13]. The molecular mechanisms underlying the abscopal effects are not completely understood. However, radiation therapy induced abscopal response appears to be synergized with the combination of targeted and immunotherapeutic agents $[14,15]$. Current evidence indicates that radiation therapy when used either alone or in combination with immunotherapeutic agents produces abscopal responses in patients with metastatic malignancies. Most of the clinical evidence of the abscopal effects of radiation therapy has been reported in the form of case reports and small non-randomized studies $[16,17]$. Abscopal effects of radiation therapy have been observed in a variety of tumor types including melanoma, renal cell, pulmonary, and hepatic cancers.

Traditionally, it has been believed that the central nervous system (CNS) was an encased almost separate organ that had protection from the rest of the body. Furthermore, the CNS had been considered to have an immune privilege $[18,19]$. Regular immune responses from therapeutic interventions were thought to be very limited in the CNS. The idea that an abscopal effect could be induced by the radiation therapy to brain would thus sound particularly unusual due to the traditional belief that the brain has its own distinctive immune microenvironment. However, emerging evidence now demonstrates that cranial radiation treatment to one or more brain metastases can induce abscopal responses in tumors away from the treated CNS metastatic sites. In this report we describe a case of an extracranial abscopal effect seen in a large chest mass when whole brain radiation therapy was used to treat a patient who had brain metastasis from melanoma,

\section{Case Report}

A 42-year-old female patient was initially diagnosed in August 2012 to have a stage-III melanoma of the right upper skin of the back. At that time, a local surgical excision was performed to remove her tumor. The pathology report indicated that the patient had positive lateral margins and tumor invasion up to $4.0 \mathrm{~mm}$. The wide local excision or sentinel lymph node staging was not performed at that time due to limitations of patient's health insurance, but the patient remained asymptomatic for over 5 years. However, in September 2017 the patient developed a large metastatic lesion in her upper right retropectoral region of the chest wall. Molecular analysis (Caris Life Sciences, Irving, TX, USA) of the initial biopsy of the chest lesions in January 2018 revealed the presence of a BRAF mutation (p.V600E) on exon 79 and RBI mutations on exon 24 (C.540-1 G>A) as well as on exon 60 (C.2490-1 G>A) (Table 1). Moreover, the initial immunohistochemistry assessment of biopsy chest lesions indicated an over expression of multiple tumor biomarkers such as excision repair crosscomplementation group 1 (ERCC 1$), 0^{6}$-methylguanineDNA-methyltransferase (MGMT) and class III $\beta$-tubulin (TUBB3) (Table 2).

Since BRAF mutations are sensitive to molecularly targeted therapies, dabrafenib therapy with a MAPK kinase (MEK) inhibitor, trametinib was recommended for the patient. Unfortunately, prior to the start of the dabrafenib and trametinib therapy, the patient developed CNS symptoms including headache, dizziness and disequilibrium. Further staging with a computerized tomography (CT) scan of the abdomen and pelvis that was performed and it revealed a cystic right ovary of a size of $3.7 \times 3.1 \mathrm{~cm}^{2}$ without any evidence of metastatic disease or regional metastasis. An MRI of the brain revealed at least eight ovoid enhancing lesions measuring up to $0.9 \mathrm{~cm}$, which were scattered throughout the bilateral cerebral hemispheres and left thalamus compatible with metastatic melanoma. No intracranial hemorrhage or other fluid was noted.

An imaging of the CT scan of the chest (Fig. 1A) in December 2017 was revealed a large right infraclavicular and right chest wall soft tissue mass measuring $10.3 \times 5.1 \mathrm{~cm}^{2}$, which was intimately, related to the neurovascular structures. A confluent mass measuring $5.4 \times 3.6 \mathrm{~cm}^{2}$ and inferior $5.6 \times 3.9 \mathrm{~cm}^{2}$ mass 
Table 1. Molecular profiling of biopsy of the metastatic chest lesions

\begin{tabular}{ccccc}
\hline Evaluation & Gene & Status & Protein alteration & Exon \\
\hline Initial biopsy & BRAF & Mutated & p.V600E & 71 \\
& RB1 & Mutated & C.540-1 G>A & 24 \\
& Mutated & C.2490-1 G $>$ A & 60 \\
Repeat biopsy & Mutated & $p . V 600 \mathrm{E}$ & 15 \\
& RBAF & Mutated & C.540-1 G>A & 6 \\
& Mutated & C.2490-1 G $>$ A & 24 \\
\hline
\end{tabular}

Table 2. Immunohistochemistry assessment of biopsy of the metastatic chest lesions

\begin{tabular}{lccccc}
\hline \multirow{2}{*}{ Biomarker } & \multicolumn{2}{c}{ Biopsy } & \multicolumn{2}{c}{ Re-biopsy } \\
\cline { 2 - 4 } \cline { 5 - 5 } \cline { 5 - 5 } ERCC1 & Status & Outcome & & Status & Positivity \\
MLH1 & Positive & $2+, 50 \%$ & & Positive & $2+, 80 \%$ \\
MSH2 & NT & NA & & Positive & $1+, 100 \%$ \\
MSH6 & NT & NA & & Positive & $1+, 100 \%$ \\
PMS2 & NT & NA & & Positive & $1+, 100 \%$ \\
TUBB3 & NT & NA & & Positive & $1+, 100 \%$ \\
PDL-1 & Positive & $3+, 90 \%$ & & Positive & $2+, 100 \%$ \\
TrK A/B/C & Negative & $0,100 \%$ & & Negative & $0,100 \%$ \\
MGMT & NT & NA & & Negative & $0,100 \%$ \\
\hline
\end{tabular}

NT, not tested; NA, not applicable.

was also visualized in the axilla (Fig. 2A). In addition, a 7.0-mm nodule was noted in the right upper outer quadrant of the breast. The chest wall otherwise appeared free of disease and the patient had a normal bone density. A bone scan showed no evidence of osseous metastasis. Since the patient had multiple brain metastases, stereotactic radiation therapy (SRS) was deemed not to be an appropriate strategy for the patient. Whole brain radiation therapy (WBRT) remains standard for patients whose brain metastasis is not amenable to SRS $[20,21]$. Therefore, the patient was administered with WBRT to a dose of 30 Gy delivered in 15 fractions to her multiple CNS lesions. Concurrently, the patient also received genetargeted therapy with systemic dabrafenib and trametinib. The basis for selecting the moderate fractionation of radiation for this patient was our previous experience that we had when we used a shorter course and higher fractionation schedule in patients who were treated with radiation and targeted therapies. The local effects of the treated tissue were exacerbated despite the use of targeted conformal approach. Therefore, we used a standard fractionation schedule to prevent the potential increased local reaction.
A repeat CT scan of the patient's chest in April 2018 revealed a significant reduction (90\%) in her chest mass with no pleural effusion (Fig. 1B). The confluent tumor mass also reduced considerably in the axilla (Fig. 2B). The residual patient's disease in the chest wall and axilla was treated with a local field to a dose of 66 Gy delivered in 33 fractions with concurrent systemic dabrafenib and trametinib therapy. This portion of the treatment was administered between March 5, 2018 and May 22, 2018. The patient's restaging bone scan after her treatment was negative for metastasis (Fig. 3). The patient had an excellent resolution of all residual disease in her chest. The patient was clinically disease free and her CNS lesions had regressed completely.

The abscopal effect was noted during the time the patient received cranial radiation therapy to her CNS and was receiving concurrent systemic gene-targeted therapy. She had a significant resolution of her large chest wall mass within 3 weeks of beginning her CNS treatment. The remainder of the chest wall lesion responded extremely well to local radiation therapy given with her gene-targeted therapy. Due to the patient's insurance delay, her radiation therapy was ended 

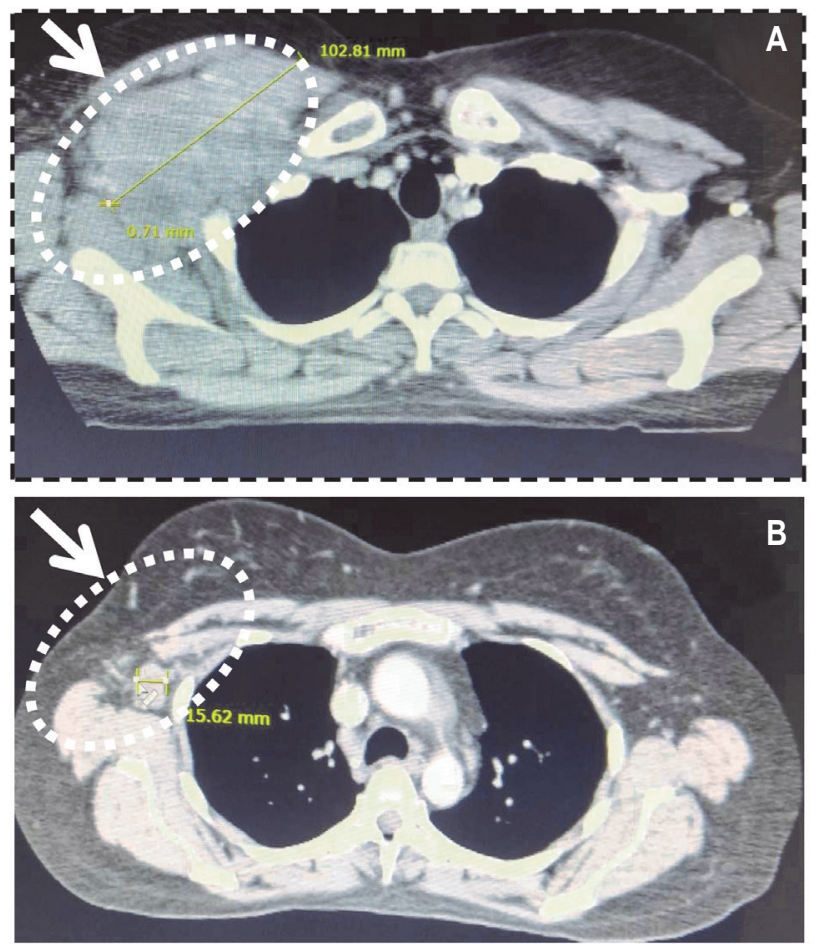

Fig. 1. Computerized tomography (CT) imaging of the chest. (A) CT scan of the chest revealing the large right infraclavicular and right chest wall soft tissue mass, which was intimately, related to the neurovascular structures prior to central nervous system (CNS) radiation therapy. (B) CT scan of the chest revealing dissipating tumor mass following CNS radiation therapy.

while continuing with dabrafenib and trametinib therapy. During the absence of her radiation therapy, the patient developed new lesions in her chest area. Molecular profiling of a repeat biopsy of her new chest lesions in July 2018 revealed the presence of several gene mutations including BRAF mutation (Table 1). Repeat immunohistochemistry assessment of her biopsy indicated an over-expression of numerous tumor biomarkers including ERCC1, MGMT, and TUBB3 (Table 2).

While continuing on dabrafenib/trametinib therapy without radiation therapy, the patient experienced recurrent disease immediately inferior to her prior treated fields that quickly became metastatic to other chest and body sites. These findings indicate the dabrafenib/trametinib therapy was not effective possibly due to a BRAF inhibitor resistance. Moreover, the findings of this case also suggest that patient experienced an initial abscopal response during cranial radiation therapy to her brain lesions. Despite continued systemic gene-targeted therapy in the absence of any further radiation therapy, the patient died due to wide spread disease recurrence outside of her treatment fields approximately 4 months later.
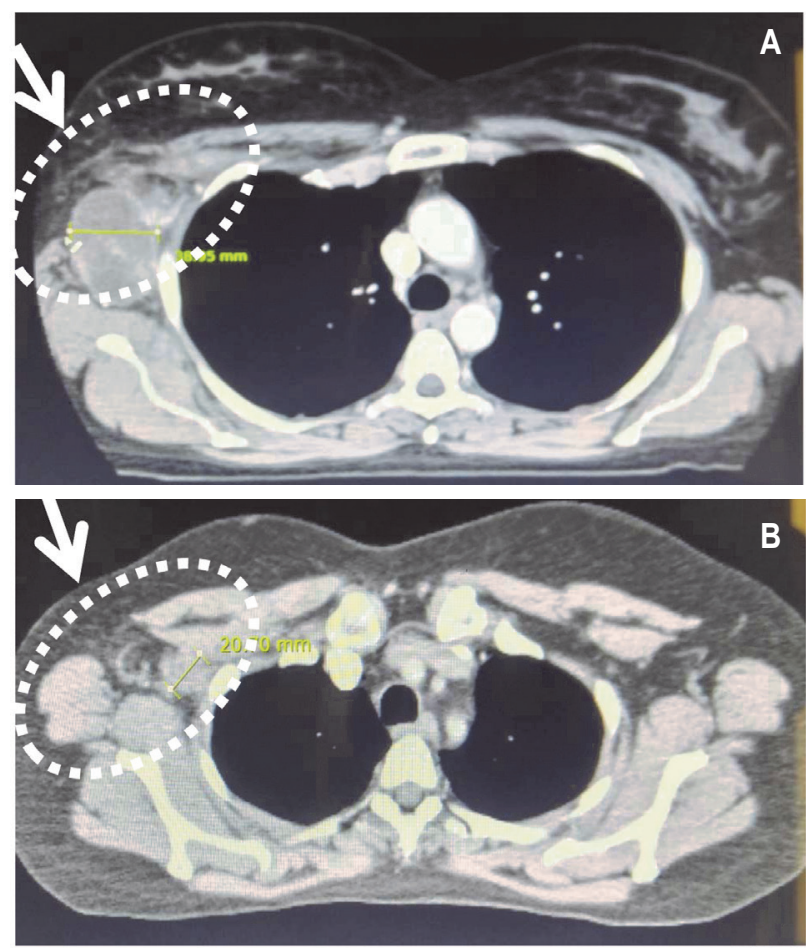

Fig. 2. Computerized tomography (CT) imaging of the axilla. (A) CT scan of the axilla showing confluent mass in axilla prior to central nervous system (CNS) radiation therapy. (B) CT scan of the axilla showing dissipating tumor following CNS radiation therapy.

\section{Discussion}

Metastatic malignant melanoma is an aggressive and often fatal disease with a median survival range from 2 to 11 months. Long-term survivorship is rare for this disease. Radiation therapy is currently the most widely adopted method to treat brain metastases of various malignancies including melanoma. Historically, the brain was thought to be distinct organ with its own immune privilege. It has been assumed that nascent tumor associated antigens produced by radiation therapy did not pass through the blood-brain barrier [22]. However, metastasis of malignant cells to the brain often implies a blood-brain barrier opening. Thus, tumor associated antigens produced during radiation therapy can cross the blood-brain barrier leading to immune modulations and subsequent abscopal effects. To date, only one other case report of melanoma has been reported in the literature, which describes this unique effect. Okwan-Duodu et al. [23] first observed an abscopal response following cranial radiation therapy in a 67-year-old male melanoma patient with brain metastasis. We are now the second investigators to observe 


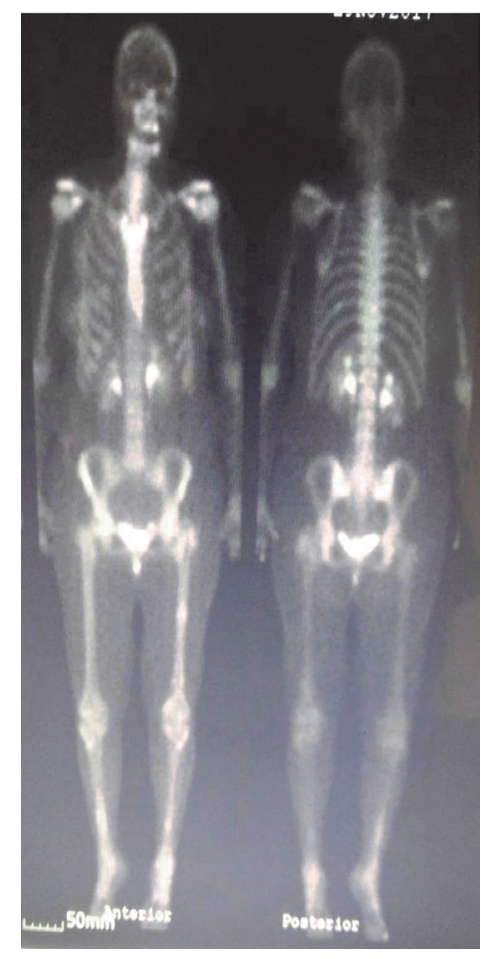

Fig. 3. A restaging bone scan of the patient showing no bone metastasis following her chest wall radiation therapy.

and report the abscopal effect induced by cranial radiation therapy in a melanoma patient with a brain metastasis.

Based on the findings of a multi-center randomized doubleblind phase III trial $[24,25]$, the combination of dabrafenib and trametinib has become as the standard targeted treatment in patients with BRAF mutation-positive melanoma. Since the patient's tumor was BRAF mutation-positive, the patient received molecularly targeted therapy using dabrafenib and trametinib after the detection of a large metastatic lesion in her upper right retropectoral region of the chest wall. Cranial radiation therapy was also administered concurrently for her CNS lesions. Three weeks after starting radiation therapy, the patient experienced a significant reduction in not only her CNS metastases but also in her upper chest wall mass indicating the abscopal effect of radiation therapy. The patient was essentially clinically disease free for a short term. When the radiation ceased and the patient received only targeted therapy, disease recurrence was noted within 12 weeks of cessation of her radiation treatment.

The disease recurrence in our case could be due to the development of resistance to BRAF/MEK inhibition by the molecularly targeted therapy with dabrafenib and trametinib or the lack of radiation therapy induced abscopal effect through the systemic immunostimulation. Multiple recent studies have demonstrated that resistance to molecularly targeted therapies is driven by multiple mechanisms functioning in concert, supporting these findings [26-28]. In metastatic melanoma patients, BRAF inhibitor resistance is supported through recovery of MAPK/Erk signaling or activation of PI3K/Akt signaling pathways. These signaling pathways may be activated through either mutations, copynumber alterations, or changes in expression of the protein markers. A multi-center analysis of BRAF inhibitor resistance in pre-treatment and post-progression patients of melanoma has identified resistance driving events in 77/132 (58.3\%) of samples obtained from 100 individuals, but failed to identify any known mechanism of resistance in the remaining 55 (41.7\%) samples [29]. Thus, studies evaluating BRAF inhibitor resistance to molecularly targeted therapies still leave an incomplete understanding of the diverse set of mechanisms supporting BRAF inhibitor resistance.

Evidence from earlier studies shows that an immunemediated response is one of the most critical mechanisms of an abscopal effect of radiation therapy. Immune-mediated responses occur when radiation induces the immunogenic cell death of the cancer cells and subsequent release of various cytokines and other signaling molecules into the circulation. Consequently, alterations occur in the tumor microenvironment leading to the influx of immune cells to the lesion sites through antigen-presenting cells (APC). Specifically, APCs recognize tumor-specific antigens released by the dead cells. Evidence suggests that two critical factors play an important role during the induction of an abscopal effect by radiation therapy. First is the generation of a sufficient number of CD8+ T cells to activate the immune system. Secondly, the production and presentation of tumor-associated antigen by APCs to CD8+ T cells which subsequently liberate specific killer $T$ cells to attack the cancer cells at the primary site and other metastatic locations. Thus, radiation therapy boosts the systemic antitumor response of the immunotherapy and enhances the probability of abscopal effects in the treatment of malignant cancers,

More recent advances in the development of molecularly targeted agents have dramatically shifted the landscape of the treatment of metastatic melanoma with improved longer survival outcomes [30-32]. The introduction of novel immune modulators such as cytotoxic T-lymphocyte antigen-4 (CTLA4) and programmed death-1 (PD-1)/programmed death ligand-1 (PDL-1) based treatments and VEGF inhibitors have been an integral part of the immunogenic tumors such as melanoma 
$[33,34]$. Nivolumab is a fully human immunoglobulin G4 immune checkpoint inhibitor antibody that targets PD-1 and promotes antitumor immunity. Specifically, it exerts an antitumor immune response by blocking the interaction between PD-1 and its ligands leading to inhibition of the PD-1 pathway. Based on the international, double blind, randomized phase III trial data [35,36], on November 23, 2015, the US Food and Drug Administration approved nivolumab as a single agent for the first-line treatment of patients with $\mathrm{BRAF}^{\mathrm{V} 600}$ mutated unresectable or metastatic melanoma. Emerging evidence from preclinical and clinical studies suggest that a combination of radiotherapy and immunotherapy augments the antitumor response much more than either therapy alone [37].

In summary, this case provides new insight into how cranial radiation therapy can induce an extracranial abscopal effect in a patient who had metastatic melanoma. As immunotherapy and targeted therapies become the mainstay treatment for metastatic melanoma, reports on their combination therapy with radiation therapy are continuing to emerge and the abscopal effects appear to become a more common finding in the treatment of immunogenic tumors including melanoma.

\section{Conflict of Interest}

No potential conflict of interest relevant to this article was reported.

\section{Acknowledgments}

The authors are thankful to Pradheeth Reddy and Sreeneha Reddy for their assistance in preparing figures and tables as well as in editing and proofreading the manuscript.

\section{References}

1. Koller KM, Mackley HB, Liu J, et al. Improved survival and complete response rates in patients with advanced melanoma treated with concurrent ipilimumab and radiotherapy versus ipilimumab alone. Cancer Biol Ther 2017;18:36-42.

2. Grimaldi AM, Simeone E, Giannarelli D, et al. Abscopal effects of radiotherapy on advanced melanoma patients who progressed after ipilimumab immunotherapy. Oncoimmunology 2014;3:e28780.

3. Eggermont AM, Robert C. New drugs in melanoma: it's a whole new world. Eur J Cancer 2011;47:2150-7.

4. Beaver JA, Theoret MR, Mushti S, et al. FDA approval of nivolumab for the first-line treatment of patients with
BRAF(V600) wild-type unresectable or metastatic melanoma. Clin Cancer Res 2017;23:3479-83.

5. Barone A, Hazarika M, Theoret MR, et al. FDA Approval Summary: pembrolizumab for the treatment of patients with unresectable or metastatic melanoma. Clin Cancer Res 2017;23:5661-5.

6. Chuk MK, Chang JT, Theoret MR, et al. FDA Approval Summary: accelerated approval of pembrolizumab for secondline treatment of Metastatic Melanoma. Clin Cancer Res 2017;23:5666-70.

7. Park $B$, Yee $C$, Lee $K M$. The effect of radiation on the immune response to cancers. Int J Mol Sci 2014;15:927-43.

8. Kumari A, Simon SS, Moody TD, Garnett-Benson C. Immunomodulatory effects of radiation: what is next for cancer therapy? Future Oncol 2016;12:239-56.

9. Mole $\mathrm{RH}$. Whole body irradiation; radiobiology or medicine? Br J Radiol 1953;26:234-41.

10. Siva S, MacManus MP, Martin RF, Martin OA. Abscopal effects of radiation therapy: a clinical review for the radiobiologist. Cancer Lett 2015;356:82-90.

11. Brix N, Tiefenthaller A, Anders H, Belka C, Lauber K. Abscopal, immunological effects of radiotherapy: narrowing the gap between clinical and preclinical experiences. Immunol Rev 2017;280:249-79.

12. Abuodeh $Y$, Venkat $P$, Kim S. Systematic review of case reports on the abscopal effect. Curr Probl Cancer 2016;40:25-37.

13. Chandra RA, Wilhite TJ, Balboni TA, et al. A systematic evaluation of abscopal responses following radiotherapy in patients with metastatic melanoma treated with ipilimumab. Oncoimmunology 2015;4:e1046028.

14. Ngwa W, Irabor OC, Schoenfeld JD, Hesser J, Demaria S, Formenti SC. Using immunotherapy to boost the abscopal effect. Nat Rev Cancer 2018;18:313-22.

15. Chicas-Sett R, Morales-Orue I, Rodriguez-Abreu D, LaraJimenez $P$. Combining radiotherapy and ipilimumab induces clinically relevant radiation-induced abscopal effects in metastatic melanoma patients: a systematic review. Clin Transl Radiat Oncol 2017:9:5-11.

16. Golden EB, Chhabra A, Chachoua A, et al. Local radiotherapy and granulocyte-macrophage colony-stimulating factor to generate abscopal responses in patients with metastatic solid tumours: a proof-of-principle trial. Lancet Oncol 2015;16:795803.

17. Hiniker SM, Reddy SA, Maecker HT, et al. A prospective clinical trial combining radiation therapy with systemic immunotherapy in metastatic melanoma. Int J Radiat Oncol Biol Phys 2016;96:578-88. 
18. Muldoon LL, Alvarez JI, Begley DJ, et al. Immunologic privilege in the central nervous system and the blood-brain barrier. J Cereb Blood Flow Metab 2013;33:13-21.

19. Negi N, Das BK. CNS: Not an immunoprivilaged site anymore but a virtual secondary lymphoid organ. Int Rev Immunol 2018;37:57-68.

20. Franchino F, Ruda R, Soffietti R. Mechanisms and therapy for cancer metastasis to the brain. Front Oncol 2018;8:161.

21. Eichler AF, Loeffler JS. Multidisciplinary management of brain metastases. Oncologist 2007;12:884-98.

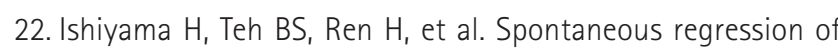
thoracic metastases while progression of brain metastases after stereotactic radiosurgery and stereotactic body radiotherapy for metastatic renal cell carcinoma: abscopal effect prevented by the blood-brain barrier? Clin Genitourin Cancer 2012;10:196-8.

23. Okwan-Duodu D, Pollack BP, Lawson D, Khan MK. Role of radiation therapy as immune activator in the era of modern immunotherapy for metastatic malignant melanoma. Am J Clin Oncol 2015;38:119-25.

24. Long GV, Eroglu Z, Infante J, et al. Long-term outcomes in patients with BRAF V600-mutant metastatic melanoma who received dabrafenib combined with trametinib. J Clin Oncol 2018;36:667-73.

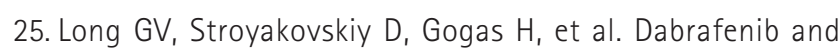
trametinib versus dabrafenib and placebo for Val600 BRAFmutant melanoma: a multicentre, double-blind, phase 3 randomised controlled trial. Lancet 2015;386:444-51.

26. Moriceau G, Hugo W, Hong A, et al. Tunable-combinatorial mechanisms of acquired resistance limit the efficacy of BRAF/ MEK cotargeting but result in melanoma drug addiction. Cancer Cell 2015;27:240-56.

27. Long GV, Fung C, Menzies AM, et al. Increased MAPK reactivation in early resistance to dabrafenib/trametinib combination therapy of BRAF-mutant metastatic melanoma. Nat Commun 2014;5:5694.

28. Wagle $N$, Van Allen EM, Treacy DJ, et al. MAP kinase pathway alterations in BRAF-mutant melanoma patients with acquired resistance to combined RAF/MEK inhibition. Cancer Discov 2014;4:61-8.
29. Johnson DB, Menzies AM, Zimmer L, et al. Acquired BRAF inhibitor resistance: a multicenter meta-analysis of the spectrum and frequencies, clinical behaviour, and phenotypic associations of resistance mechanisms. Eur J Cancer 2015;51:2792-9.

30. Long GV, Flaherty KT, Stroyakovskiy D, et al. Dabrafenib plus trametinib versus dabrafenib monotherapy in patients with metastatic BRAF V600E/K-mutant melanoma: long-term survival and safety analysis of a phase 3 study. Ann Oncol 2017;28:1631-9.

31. Lim SY, Menzies AM, Rizos H. Mechanisms and strategies to overcome resistance to molecularly targeted therapy for melanoma. Cancer 2017;123(S11):2118-29.

32. Ascierto PA, McArthur GA, Dreno B, et al. Cobimetinib combined with vemurafenib in advanced BRAF(V600)mutant melanoma (coBRIM): updated efficacy results from a randomised, double-blind, phase 3 trial. Lancet Oncol 2016;17:1248-60.

33. Rausch MP, Hastings KT. Immune checkpoint inhibitors in the treatment of melanoma: from basic science to clinical application. In: Ward WH, Farma JM, editors. Cutaneous melanoma: etiology and therapy. Brisbane, Australia: Codon Publications; 2017.

34. Ott PA, Hodi FS, Buchbinder El. Inhibition of immune checkpoints and vascular endothelial growth factor as combination therapy for metastatic melanoma: an overview of rationale, preclinical evidence, and initial clinical data. Front Oncol 2015;5:202.

35. Weber JS, D'Angelo SP, Minor D, et al. Nivolumab versus chemotherapy in patients with advanced melanoma who progressed after anti-CTLA-4 treatment (CheckMate 037): a randomised, controlled, open-label, phase 3 trial. Lancet Oncol 2015;16:375-84.

36. Hazarika M, Chuk MK, Theoret MR, et al. U.S. FDA Approval Summary: nivolumab for treatment of unresectable or metastatic melanoma following progression on ipilimumab. Clin Cancer Res 2017;23:3484-8.

37. Ko EC, Formenti SC. Radiotherapy and checkpoint inhibitors: a winning new combination? Ther Adv Med Oncol 2018 Apr 11 [Epub]. http://doi.org/10.1177/1758835918768240. 